\section{(2) OPEN ACCESS}

\title{
Prognostic value of C-reactive protein in adults with congenital heart disease
}

\author{
Laurie W Geenen, Vivan J M Baggen, Annemien E van den Bosch (D) , \\ Jannet A Eindhoven, Robert M Kauling, Judith A A E Cuypers, \\ Jolien W Roos-Hesselink (D) , Eric Boersma
}

- Additional material is published online only. To view please visit the journal online (http://dx.doi.org/10.1136/ heartjnl-2020-316813).

Cardiology, Erasmus University Medical Center Rotterdam, Rotterdam, The Netherlands

\section{Correspondence to} Dr Jolien W Roos-Hesselink, Cardiology, Erasmus MC, Rotterdam 3000 CA, The Netherlands:

j.roos@erasmusmc.nl

Received 28 February 2020 Revised 8 September 2020 Accepted 10 September 2020 Published Online First 15 October 2020

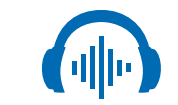

Listen to Podcast heart.bmj.com
Check for updates

(C) Author(s) (or their employer(s)) 2021. Re-use permitted under CC BY-NC. No commercial re-use. See rights and permissions. Published by BMJ.

To cite: Geenen LW, Baggen VJM, van den Bosch AE, et al. Heart 2021;107:474-481.

\section{ABSTRACT \\ Background High-sensitivity $C$ reactive protein} (hs-CRP) has been associated with outcomes in adult congenital heart disease (ACHD). However, its prognostic value beyond $\mathrm{N}$-terminal pro $\mathrm{B}$ type natriuretic peptide (NT-proBNP) or troponin T remains unknown. We studied the temporal evolution of hs-CRP, as well as the relation between hs-CRP and adverse clinical outcomes independent of NT-proBNP and troponin T in patients with ACHD.

Methods In this prospective cohort study, we enrolled 602 patients with ACHD (2011-2013) who underwent baseline and thereafter annual blood sampling during 4 years. Hs-CRP, hs-troponin T and NT-proBNP were measured. The primary endpoint was composed of death or heart failure (HF). Cox regression and Joint Modelling was used to relate $2 \log$ hs-CRP levels with the endpoint, with adjustment for baseline characteristics and (repeated) hs-troponin T and NT-proBNP measurements. Results Hs-CRP was measured at baseline in 591 patients, median age 33 years, 58\% men, 90\% New York Heart Association I with an average of 4.3 measurements per patient. Median follow-up was 5.9 (IQR 5.3-6.3) years (99.2\% complete) and 69 patients met the endpoint. Higher baseline hs-CRP was independently associated with higher risk of death or HF (HR 1.36, $95 \% \mathrm{Cl} 1.19$ to 1.55$)$. Hs-CRP increased over time prior to death or HF, and repeated hs-CRP measurements were associated with the endpoint, independent of repeated NT-proBNP and hs-troponin T (HR 1.54, 95\% Cl 1.24 to 1.98).

Conclusions Hs-CRP carries incremental prognostic value for the risk of death or HF, beyond NT-proBNP and hs-troponin T. Hs-CRP increased prior to the occurrence of HF or death, supporting the role of inflammation in the clinical deterioration of patients with ACHD.

\section{INTRODUCTION}

Adults with congenital heart disease (ACHD) are a growing patient population with a high healthcare utilisation. ${ }^{12}$ Despite the increased survival prospects, patients with ACHD carry an increased risk of heart failure (HF), arrhythmias, reinterventions and early demise. ${ }^{3-5}$ Although mechanisms such as chronic pressure or volume overload that can lead to cardiac deterioration are well described in patients with $\mathrm{ACHD},{ }^{6}$ the potential role of chronic inflammation has been investigated to a lesser extent. A recent study by Opotowsky et al demonstrated that higher high-sensitivity C-reactive protein (hsCRP) was associated with higher risk of cardiovascular events and mortality in patients with ACHD. ${ }^{7}$ These findings suggest that chronic inflammation may contribute to the pathophysiology of deterioration in $\mathrm{ACHD}$, as hs-CRP is a sensitive marker of systemic inflammation.

Currently, it is unknown how hs-CRP relates to other biomarkers and echocardiographic measures in ACHD. Besides the great interest in the pathophysiological mechanism of hs-CRP, the additive prognostic value of hs-CRP beyond conventional biomarkers, such as N-terminal pro B type natriuretic peptide (NT-proBNP) and high-sensitivity troponin-T (hs-TnT), has not yet been assessed. It is therefore unclear how hs-CRP could enhance risk stratification. Moreover, it is unknown how hs-CRP evolves over time. Studying these temporal patterns may contribute to better understanding of hs-CRP release in patients with ACHD.

The aim of this study was to investigate the association of hs-CRP with other biomarkers and echocardiographic measurements, as well as its association with long-term cardiovascular outcomes in patients with ACHD. Moreover, we studied the temporal evolution of hs-CRP and the relation of these evolutions with the risk of cardiovascular events.

\section{METHODS}

\section{Patient and public involvement}

Patients or the public were not involved in the design, or conduct, or reporting, or dissemination plans of our research.

\section{Study design and population}

This is a prospective observational single-centre cohort study, including 602 clinically stable patients with moderately or severely complex ACHD. Detailed aspects of the study protocol have been described previously. ${ }^{8} 9$ Patients were enrolled during a routine visit at the outpatient clinic (20112013). Exclusion criteria were age $<18$ years, mild ACHD, renal impairment (creatinine $>200$ $\mu \mathrm{mol} / \mathrm{L}$ ) or pregnancy. All patients underwent physical examination by a cardiologist, ECG, echocardiography and venous blood sampling at the day of enrolment. Thereafter, yearly outpatient visits were scheduled for 4 years and venous blood sampling was repeated. Patient management was according 
to the discretion of the treating physician, based on prevailing guidelines. $^{1011}$

The study was performed according to the principles outlined in the Declaration of Helsinki. All patients gave written informed consent for their participation in the study.

\section{Laboratory testing}

During baseline and follow-up study visits, peripheral venous blood sampling was performed. Blood samples were directly used, or stored at $-80^{\circ} \mathrm{C}$ in the biobank until batch analysis was performed. NT-proBNP and estimated glomerular filtration rate (eGFR) were directly measured in fresh blood samples in our clinical chemistry laboratory. Serum hs-CRP and hs-TnT were measured batch wise from the same samples (first batches of 591 baseline measurements in 2012; second batches of 1943 follow-up samples in 2018) in thawed serum samples in the same laboratory using Roche immunoturbidimetric assays and electrochemiluminescence immunoassays, respectively (Roche Diagnostics, Basel, Switzerland). Samples had not been exposed to a prior freeze-thaw cycle. On average 4.3 measurements per patient were available. The lower limits of detection of hs-CRP, hs-TnT and NT-proBNP were $0.30 \mathrm{mg} / \mathrm{L}, 5 \mathrm{ng} / \mathrm{L}$ and $0.6 \mathrm{pmol} / \mathrm{L}$, respectively. Lower limit of blank of hs-TnT was $3 \mathrm{ng} / \mathrm{L}$. For analytical purposes, hs-CRP levels $<0.30 \mathrm{mg} / \mathrm{L}$ were substituted with $0.15 \mathrm{mg} / \mathrm{L}$, and hs-TnT levels $<3 \mathrm{ng} / \mathrm{L}$ were substituted with $1.5 \mathrm{ng} / \mathrm{L}$. Elevated levels of hs-CRP and NT-proBNP were defined as; $>3.0 \mathrm{mg} / \mathrm{L}$ and $>14 \mathrm{pmol} / \mathrm{L}$, respectively.

\section{Definition and assessment of endpoints}

The primary endpoint was defined as all-cause mortality or HF (defined as HF requiring initiation/intensification of HF medication or requiring hospitalisation). The secondary endpoint was defined as any major adverse cardiovascular event (MACE) and was composed of; all-cause mortality, HF, hospitalisation for cardiac reasons (eg, endocarditis), arrhythmia (symptomatic and recorded, or requiring treatment), thromboembolic events or cardiac (re)interventions (surgical or percutaneous). Survival status was checked in the Municipal Population Register. Endpoint events were adjudicated by two investigators who were blinded for any study related information.

\section{Statistical analysis}

Sample size calculation was performed and has been previously been described. ${ }^{9}$ In the current study, the composite endpoint of death or HF was considered the primary endpoint because of its greater clinical relevance than any MACE.

Continuous data are presented as mean \pm SD or median (IQR). Trends between clinical characteristics and hs-CRP tertiles were tested with the $\chi^{2}$ Mantel-Haenszel test for trend or linear regression. Skewed biomarker distributions were transformed by taking logarithms to the base 2 . The variability of hs-CRP and reference change value (RCV) were assessed in primary endpoint-free patients. The methods are specified in online supplemental file 1 .

Survival curves were derived using the Kaplan-Meier method and were stratified based on elevated NT-proBNP and hs-CRP levels. The log-rank test was used to compare survival curves. Cox regression analyses were performed to investigate the association between baseline hs-CRP and the endpoints. We present crude HRs, and HRs that are adjusted for NT-proBNP, hs-TnT and baseline characteristics including age, sex, congenital diagnosis, New York Heart Association (NYHA) class, cardiac medication and systemic ventricular function.
The temporal evolution of hs-CRP was described using linear mixed effect models (LME) and Cox models were used to assess the association between hs-CRP and the endpoints. The LME model is described in online supplemental file 2. Parameters of LME and Cox models were estimated jointly in Joint Models to relate biomarker trajectories with endpoints and avoid bias. ${ }^{12}$ The rational and assumptions of Joint Models are outlined in online supplemental file 3. Hs-CRP measurements that were taken after occurrence of endpoints were discarded in the analyses. We reported crude HRs, and HRs adjusted for repeatedly measured NT-proBNP and hs-TnT, ${ }^{13}$ and baseline characteristics. Subanalyses were performed to assess the association between hs-CRP and endpoints in patients with elevated baseline NT-proBNP. The C-index was calculated to assess the discriminative value of hs-CRP.

Missing baseline covariates were handled by single imputation. SPSS (V.24) and R statistical software (V.3.6.1, packages mice, Survival, nlme, JMbayes) were used. A two-tailed $\mathrm{p}<0.05$ was considered statistically significant.

\section{RESULTS}

\section{Baseline characteristics}

In 591 patients (98.2\%), hs-CRP was measured at baseline and was therefore included in the current analysis. Median age was 33 (IQR 25-41) years, 58\% of the patients were men and 90\% were NYHA class I (table 1). The most prevalent congenital diagnoses were: Tetralogy of Fallot $(n=174,29 \%)$, aortic stenosis $(n=136$, $23 \%)$ and aortic coarctation $(n=110,19 \%)$. Median hs-CRP level was 1.50 (IQR 0.60-3.50) mg/L. Hs-CRP was below the limit of detection in 44 patients (7.4\%), and 164 patients $(28 \%)$ had elevated hs-CRP. Higher hs-CRP was associated with older age, female sex, higher body mass index (BMI), cardiac medication use and NYHA class. Highest hs-CRP levels were found in patients with a functionally univentricular heart and those with pulmonary arterial hypertension (PAH) (figure 1). None of the echocardiographic measurements were related to hs-CRP, except for measures of left ventricular diastolic function (E/A ratio, E' wave and E/E' ratio). Hs-CRP showed significant, but weak, correlations with NT-proBNP (Spearman $\mathrm{r}=0.28, \mathrm{p}<0.001$ ) and eGFR (Spearman $\mathrm{r}=-0.14, \mathrm{p}=0.001$, but not with hs-TnT (Spearman $\mathrm{r}=0.04, \mathrm{p}=0.323$ ).

\section{Follow-up and associations between baseline hs-CRP and endpoints}

Follow-up data were complete in 586 patients (99.2\%). Median follow-up was 5.9 (IQR 5.3-6.3) years. Death or HF occurred in 69 patients $(11.8 \%)$ and MACE in 228 patients $(38.9 \%)$. Occurrence of individual endpoint events was as follows: 25 deaths, $59 \mathrm{HF}, 127$ arrhythmias, 180 hospitalisations (of which $\mathrm{n}=13$ endocarditis), 137 (re)interventions and 29 thromboembolic events. Patients in the first hs-CRP tertile $(<0.80 \mathrm{mg} / \mathrm{L})$ had a significant better HF-free and MACE-free survival than patients in higher tertiles (online supplemental file 4). Analysed continuously, hs-CRP was associated with death or HF (HR $1.36,95 \%$ CI 1.19 to 1.55 , C-index 0.66), also after adjustment for baseline NT-proBNP, hs-TnT and baseline clinical characteristics (table 2). Hs-CRP was also associated with death and HF when analysed as separate events (online supplemental material 5).

Patients with both elevated baseline hs-CRP and NT-proBNP, had the worst HF-free survival (figure 2). 
Table 1 Baseline characteristics of the study cohort for all patients and categorised according to the tertile distribution of hs-CRP

\begin{tabular}{|c|c|c|c|c|c|c|}
\hline & All & $\begin{array}{l}\mathrm{Hs}-\mathrm{CRP} \\
<0.80 \mathrm{mg} / \mathrm{L}\end{array}$ & $\begin{array}{l}\text { Hs-CRP } \\
0.80-2.50 \mathrm{mg} / \mathrm{L}\end{array}$ & $\begin{array}{l}\mathrm{Hs}-\mathrm{CRP} \\
>2.50 \mathrm{mg} / \mathrm{L}\end{array}$ & $P$ value & $\%$ missing \\
\hline Number of patients & 591 & 200 & 200 & 191 & & \\
\hline \multicolumn{7}{|l|}{ Clinical characteristics } \\
\hline Age, years & $33(25-41)$ & $31(23-40)$ & $33(25-42)$ & $34(26-42)$ & 0.002 & 0.0 \\
\hline Sex, men & $343(58)$ & $139(70)$ & $120(60)$ & $84(44)$ & $<0.001$ & 0.0 \\
\hline Surgical repair & $537(91)$ & $184(92.0)$ & $185(92.5)$ & $168(88.0)$ & 0.248 & 0.0 \\
\hline Age at surgical repair, years & $3.9(0.8-12.0)$ & $2.9(0.7-11.3)$ & $4.1(1.0-14.4)$ & $4.2(0.8-11.2)$ & 0.730 & 0.0 \\
\hline Mechanical valve & $62(9)$ & $13(7)$ & $27(14)$ & $22(12)$ & 0.063 & 0.0 \\
\hline Pacemaker & $37(3)$ & $6(3)$ & $16(8)$ & $15(8)$ & 0.065 & 0.0 \\
\hline ICD & $20(3)$ & $6(3)$ & $8(4)$ & $6(3)$ & 0.837 & 0.0 \\
\hline Cardiac medication use* & $211(36)$ & $48(24)$ & $83(42)$ & $80(42)$ & $<0.001$ & 0.0 \\
\hline Body mass index, $\mathrm{kg} / \mathrm{m}^{2}$ & $24.8 \pm 4.4$ & $23.3 \pm 3.8$ & $24.7 \pm 3.8$ & $26.4 \pm 5.0$ & $<0.001$ & 0.5 \\
\hline Heart rate, beats/minute & $74 \pm 13.4$ & $73 \pm 13$ & $71 \pm 13$ & $77 \pm 14$ & 0.016 & 1.4 \\
\hline Systolic blood pressure, $\mathrm{mm} \mathrm{Hg}$ & $126 \pm 16$ & $126 \pm 16$ & $125 \pm 15$ & $128 \pm 18$ & 0.175 & 1.9 \\
\hline 02 saturation $<90 \%$ & $17(3)$ & $4(2)$ & $4(2)$ & $9(5)$ & 0.114 & 7.4 \\
\hline NYHA class, II or III & $61(10)$ & $15(8)$ & $19(10)$ & $27(14)$ & 0.032 & 0.0 \\
\hline \multicolumn{7}{|l|}{ Electrocardiography } \\
\hline Rhythm & & & & & $0.360 t$ & 0.0 \\
\hline Sinus & $510(86)$ & $178(89)$ & $171(86)$ & $161(84)$ & & \\
\hline Paced & 44 & $10(5)$ & $18(9)$ & $16(8)$ & & \\
\hline Atrial fibrillation & 15 & $3(1)$ & $6(3)$ & $6(5)$ & & \\
\hline Other & 22 & $9(5)$ & $5(2)$ & $8(4)$ & & \\
\hline QRS duration, ms & $112(100-137)$ & $114(101-144)$ & $112(101-134)$ & $111(97-135)$ & 0.124 & 7.4 \\
\hline \multicolumn{7}{|l|}{ Echocardiography } \\
\hline Left atrial volume, $\mathrm{mL} / \mathrm{m}^{2} \ddagger$ & $20.7(15.7-29.2)$ & $21.5(14.9-27.9)$ & $20.2(16.1-30.8)$ & $20.5(16.3-28.6)$ & 0.881 & 28.4 \\
\hline LV end-diastolic volume, $\mathrm{mL} / \mathrm{m}^{2} \ddagger$ & $63.4 \pm 18.8$ & $62.3 \pm 15.8$ & $66.5 \pm 21.8$ & $61.2 \pm 18.0$ & 0.737 & 32.1 \\
\hline LV ejection fraction, $\% \neq$ & $56.0 \pm 7.8$ & $56.7 \pm 7.0$ & $55.7 \pm 7.9$ & $55.7 \pm 8.6$ & 0.305 & 32.3 \\
\hline Right ventricular fractional area change, $\%$ & $38.2 \pm 11.3$ & $38.6 \pm 10.8$ & $38.4 \pm 12.0$ & $37.7 \pm 11.2$ & 0.511 & 35.7 \\
\hline Systemic ventricular function & & & & & 0.408 & 0.0 \\
\hline Normal & $298(50)$ & $107(53)$ & $95(48)$ & $96(50)$ & & \\
\hline Mildly impaired & $206(35)$ & $69(35)$ & $69(35)$ & $68(36)$ & & \\
\hline Moderately impaired & $69(12)$ & $21(10)$ & $26(13)$ & $22(11)$ & & \\
\hline Severely impaired & $18(3)$ & $3(2)$ & $10(5)$ & $5(3)$ & & \\
\hline E/A ratio & $1.6 \pm 0.7$ & $1.7 \pm 0.6$ & $1.7 \pm 0.8$ & $1.5 \pm 0.6$ & 0.023 & 27.1 \\
\hline$E^{\prime}$ wave, $\mathrm{m} / \mathrm{s}$ & $8.2 \pm 2.6$ & $8.5 \pm 2.4$ & $8.3 \pm 2.8$ & $7.9 \pm 2.6$ & 0.039 & 34.5 \\
\hline E/E' ratio & $11.6 \pm 5.1$ & $10.8 \pm 4.1$ & $11.6 \pm 5.3$ & $12.5 \pm 5.7$ & 0.007 & 35.7 \\
\hline Severe valvular dysfunction§ & $84(14.5)$ & $25(13)$ & $23(12)$ & $36(19)$ & 0.068 & 1.9 \\
\hline \multicolumn{7}{|l|}{ Laboratory results } \\
\hline eGFR, $\mathrm{mL} / \mathrm{min} / 1.73 \mathrm{~m}^{2}$ & $90(82-90)$ & $90(86-90)$ & $90(81-90)$ & $90(80-90)$ & 0.002 & 1.5 \\
\hline Haemoglobin, mmol Fe/L & $9.22 \pm 0.99$ & $9.39 \pm 0.83$ & $9.21 \pm 0.94$ & $9.06 \pm 1.15$ & 0.001 & 1.5 \\
\hline NT-proBNP, pmol/L & $15(7-33)$ & $10(5-22)$ & $15(7-37)$ & $24(11-40)$ & $<0.001$ & 0.7 \\
\hline $\mathrm{Hs-TnT,ng/L}$ & $4.3(1.5-7.2)$ & $4.0(1.5-6.5)$ & $4.6(1.5-7.6)$ & $4.3(1.5-7.6)$ & 0.068 & 0.3 \\
\hline
\end{tabular}

Categorical variables are presented as number $(\%)$ and continuous variables as mean \pm SD or median (IQR).

*Angiotensin-converting-enzyme inhibitors, angiotensin 2 receptor blockers, beta-blocker, diuretics, calcium blockers or anti-arrhythmic drugs.

tP value for comparison of sinus rhythm vs no sinus rhythm.

¥Left-sided volumes were not measured in patients with a systemic right ventricle, univentricular heart, pulmonary hypertension or a poor acoustic window.

§Defined as maximal aortic or pulmonary valve velocity $>4.0 \mathrm{~m} / \mathrm{s}$; grade 3 or 4 out of 4 aortic, pulmonary or mitral valve regurgitation; or grade 4 out of 4 tricuspid valve regurgitation.

eGFR, estimated glomerular filtration rate; hs-CRP, high-sensitivity C-reactive protein; hs-TnT, high-sensitivity troponin T; LV, left ventricular; NT-proBNP, N-terminal pro B-type natriuretic peptide; NYHA, New York Heart Association.

\section{Associations between repeated hs-CRP and endpoints}

In total, 2421 hs-CRP measurements were available prior to the primary endpoint. Baseline hs-CRP was higher in patients with the primary endpoint $(2.53 \mathrm{mg} / \mathrm{L})$ than those who remained endpoint-free $(1.29 \mathrm{mg} / \mathrm{L})(\mathrm{p}<0.001)$. Hs-CRP further increased in anticipation to death or incident HF, whereas endpoint-free patients showed stable hs-CRP levels throughout follow-up (figure 3). The average 4-year hs-CRP increase in patients with the primary endpoint was $3.09 \mathrm{mg} / \mathrm{L}$, vs $0.07 \mathrm{mg} / \mathrm{L}$ in stable patients. In relation to MACE, no difference was found in hs-CRP evolution.

Any time during follow-up, each twofold higher hs-CRP was associated with a 2.14 times higher hazard of mortality or HF (C-index 0.82) (table 3). This remained significant after adjustment for repeatedly measured NT-proBNP and hs-TnT, and baseline clinical characteristics. Repeated hs-CRP showed 


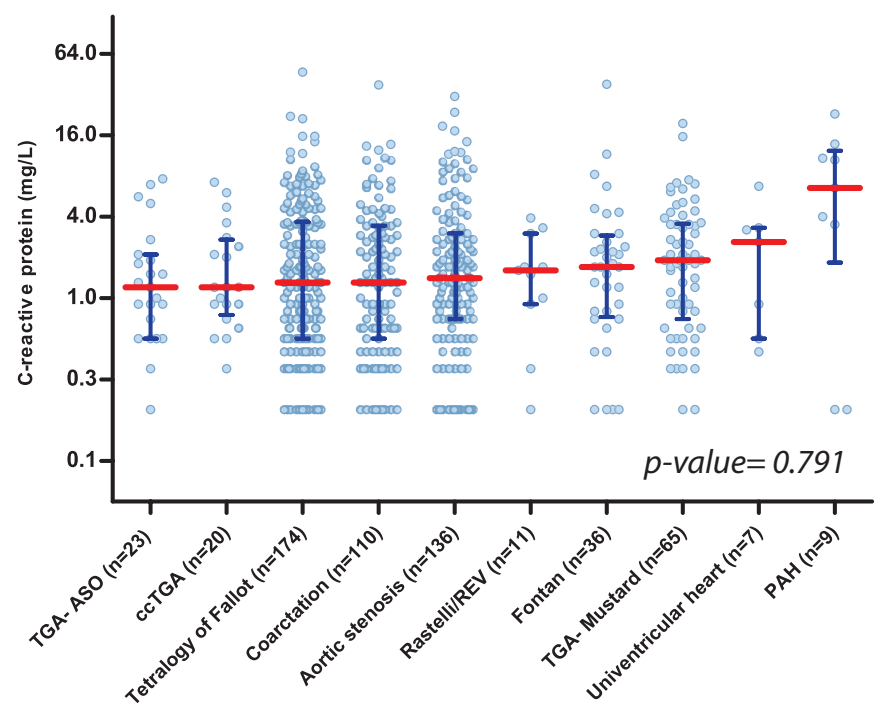

Figure 1 Hs-CRP levels according to congenital diagnosis. The median hs-CRP level in each diagnosis group is indicated by the horizontal red line together with the 25 th and 75 th percentile presented by the blue bars. $P$ value is given for comparison of hs-CRP levels between diagnoses (for the analysis univentricular heart was combined with Fontan, and Rastelli/REV with PAH). ccTGA, congenitally corrected transposition of the great arteries; hs-CRP, high-sensitivity $C$ reactive protein; $\mathrm{PAH}$, pulmonary arterial hypertension; TGA-ASO, transposition of the great arteries corrected by the arterial switch operation; TGAMustard, transposition of the great arteries corrected by the Mustard procedure.

a significant association with MACE, but not when adjusted for baseline characteristics, or other repeatedly measured biomarkers. Associations between repeated hs-CRP and individual endpoints are shown in online supplemental file 5.

As posthoc sensitivity analysis, we repeated the analysis after excluding patient with baseline hs-CRP levels $>10 \mathrm{mg} / \mathrm{L}$ $(n=34)$. This did not result in qualitative meaningful differences in strengths of the associations. Similarly, analysis excluding women who became pregnant during follow-up $(n=31)$ did not lead to different results. Posthoc analysis stratifying for sex showed that repeated hs-CRP was more strongly associated with the risk of death or HF in men than in women (HR 3.95 (95\% CI 2.39 to 8.25 ) vs 1.55 (95\% CI 1.20 to 2.03$), \mathrm{p}=0.005)$. This difference was not present when adjusted for NT-proBNP (HR
1.81 (95\% CI 1.24 to 2.78 ) vs 1.51 (95\% CI 1.17 to 1.99 ), $\mathrm{p}=0.157)$. Subanalysis restricted to patients with elevated baseline NT-proBNP demonstrated that the association between repeatedly measured hs-CRP and the risk of death or HF, is also present in these patients (online supplemental file 6).

\section{Biological variation of hs-CRP}

Based on repeated hs-CRP measurements in primary endpointfree patients, the within-subject and between-subject variation of hs-CRP were $52 \%$ and $113 \%$, respectively. The index of individuality was 0.48 , indicating that it may be preferred to have a patient-based reference value based on previously measured hs-CRP levels of the individual patient. The RCV was $149 \%$ (lognormal RCV limits $-75 \%$ to $304 \%$ ).

\section{DISCUSSION}

This study demonstrated that in patients with ACHD higher baseline hs-CRP is associated with a higher risk of death of HF, independent of NT-proBNP and hs-TnT. Furthermore, annually repeated hs-CRP measured up to 4 years, showed that on average hs-CRP levels steadily increased prior to the occurrence of death or HF and remained stable in HF-free patients. As repeated hs-CRP measurements provided prognostic information beyond repeated NT-proBNP and hs-TnT measurements, hs-CRP is a valuable potential biomarker for risk stratification in patients with ACHD.

\section{Comparison with previous studies}

In 2018, Opotowsky et al described hs-CRP in a prospective cohort consisting of 707 patients with ACHD. ${ }^{7}$ The proportion of patients with elevated hs-CRP $(>3 \mathrm{mg} / \mathrm{L})$ in our study was comparable to their study ( $28 \%$ vs $25 \%$ ), as were study settings on most aspects, except for our cohort not including mild ACHD. This might explain why we found slightly higher hs-CRP levels (median 1.50 vs $1.22 \mathrm{mg} / \mathrm{L}$ ), despite that our patients were younger, more often NYHA class I and had a lower BMI.

Whereas in our study baseline hs-CRP primarily showed an independent association with all-cause mortality and HF, Opotowsky et al found associations with other adverse cardiac events such as arrhythmia and thromboembolic events. An explanation could be the lower event rate and longer follow-up in our cohort, creating a longer time interval between baseline hs-CRP and event occurrence, probably diluting associations. The fact that repeatedly measured hs-CRP was associated with

Table 2 Association between baseline hs-CRP levels and the endpoints, adjusted for clinical characteristics, NT-proBNP and hs-TnT

\begin{tabular}{|c|c|c|c|c|}
\hline & \multicolumn{2}{|l|}{ Death or HF $(n=69)$} & \multicolumn{2}{|l|}{ MACE $(n=228)$} \\
\hline & $\mathrm{HR}(95 \% \mathrm{Cl})$ & $P$ value & $\mathrm{HR}(95 \% \mathrm{Cl})$ & $P$ value \\
\hline Baseline hs-CRP, mg/L (unadjusted) & 1.36 (1.19 to 1.55$)$ & $<0.001$ & $1.08(1.00$ to 1.16$)$ & 0.038 \\
\hline \multicolumn{5}{|l|}{ Adjusted for: } \\
\hline Baseline NT-proBNP & 1.19 (1.04 to 1.37$)$ & 0.014 & 1.00 (0.92 to 1.07$)$ & 0.910 \\
\hline Baseline hs-TnT & 1.35 (1.18 to 1.56$)$ & $<0.001$ & $1.08(1.00$ to 1.16$)$ & 0.059 \\
\hline Baseline characteristics* & 1.21 (1.05 to 1.40$)$ & 0.008 & $1.02(0.95$ to 1.10$)$ & 0.587 \\
\hline Baseline characteristics* and NT-proBNP & 1.18 (1.03 to 1.35$)$ & 0.016 & 1.00 (0.93 to 1.08$)$ & 0.989 \\
\hline Baseline characteristics*and hs-TnT & 1.18 (1.03 to 1.35$)$ & 0.018 & $1.02(0.95$ to 1.10$)$ & 0.594 \\
\hline Baseline characteristics ${ }^{*} \mathrm{NT}$-proBNP and hs-TnT & 1.17 (1.02 to 1.34$)$ & 0.023 & $1.00(0.93$ to 1.08$)$ & 0.987 \\
\hline
\end{tabular}

HRs are expressed per twofold higher biomarker level.

${ }^{*}$ Age, sex, congenital diagnosis (aortic stenosis, aortic coarctation, transposition of the great arteries operated by arterial switch procedure vs the other diagnoses), NYHA (NYHA

I vs NYHA II/III), cardiac medication use (no vs yes), systemic ventricular function (0-3).

HF, heart failure; hs-CRP, high-sensitivity C reactive protein; hs-TnT, high-sensitivity troponin T; MACE, major adverse cardiac event; NT-proBNP, N-terminal pro B type natriuretic peptide. 

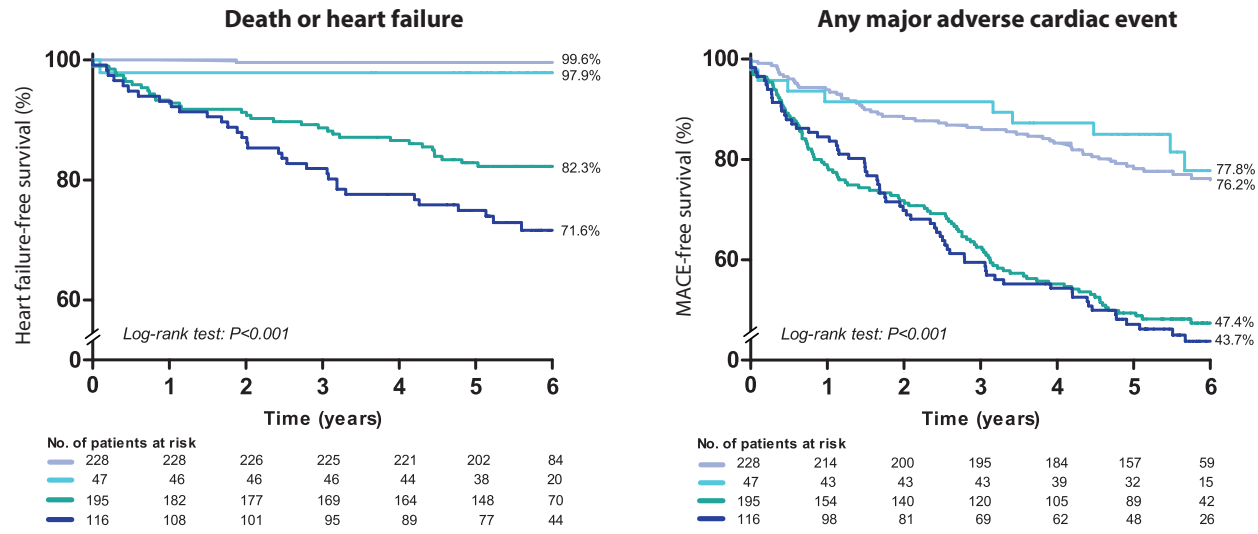

Figure 2 Heart failure-free and MACE-free survival according to normal or elevated baseline levels of hs-CRP and NT-proBNP. Elevated hs-CRP was defined as $>3 \mathrm{mg} / \mathrm{L}$ and elevated NT-proBNP as $>14$ pmol/L. hs-CRP, high-sensitivity C reactive protein; MACE, major adverse cardiovascular event; NT-proBNP; N-terminal pro B type natriuretic peptide.

arrhythmia, reintervention and hospitalisation in our study may support this, as hs-CRP will be measured more closely to the event and therefore better reflects a patients' disease status.

Scognamiglio et al investigated serial CRP measurements retrospectively in 225 adults with $\mathrm{PAH}$ due to congenital heart disease. Higher CRP correlated weakly with higher BNP, similar to our study, and was associated a higher mortality risk. While they excluded CRP measurements in patients with signs of active infection, elevated CRP levels ( $>10 \mathrm{mg} / \mathrm{L}$ ) were still measured in $54 \%$ of the patients at least once during follow-up. ${ }^{14}$ Four out of nine patients with PAH in our study had baseline hs-CRP $>10 \mathrm{mg} / \mathrm{L}$; one patient had a cold without fever; in the others, no signs of infection were present. Therefore, elevated hs-CRP seem not restricted to acute infections but may be triggered by their disease state linked to PAH.

\section{Role of inflammation in ACHD}

Hs-CRP is an acute phase protein and is commonly used blood test to assess presence of acute inflammation. Improved hs-CRP assays now allow for assessment of chronic, low inflammatory levels in not eminently inflammatory conditions, like ACHD. Findings of our study support evidence regarding chronic inflammation accompanying cardiac deterioration and mortality in patients with ACHD. ${ }^{74} 15$ The question remains whether inflammation induces HF or is rather an 'innocent bystander' and thereon reflects disease severity. Hs-CRP was not associated with measures of right or left ventricular systolic function in our study, while hs-CRP was associated with measures of diastolic dysfunction. This could be explained by age as contributing factor to both increasing hs-CRP and diastolic dysfunction. ${ }^{16}$ Nevertheless, other biomarkers that are considered more linked to the HF pathophysiology, such as NT-proBNP and hs-TnT, seem to relate to echocardiographic measurements of cardiac function more closely. ${ }^{91718}$ In addition, hs-CRP did not correlate with hs-TnT and only weakly with NT-proBNP. Therefore, causal involvement of hs-CRP in the pathophysiology of cardiac deterioration seems less plausible.

\section{Death or heart failure}

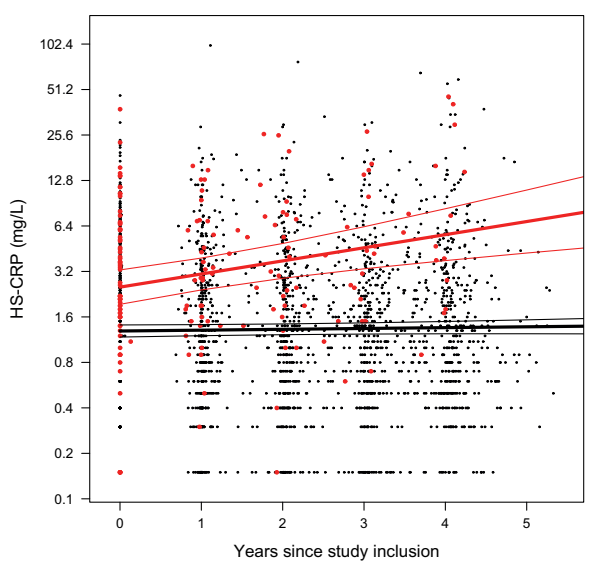

MACE

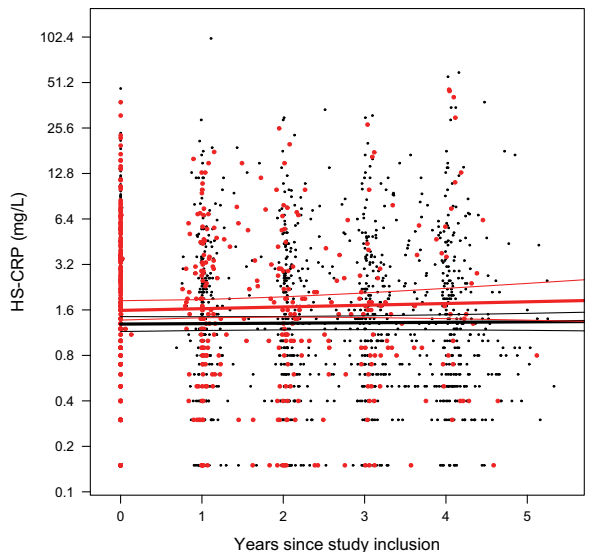

- Hs-CRP measurement in patients with the endpoint _- Average temporal evolution of hs-CRP in patients with the endpoint

- Hs-CRP measurement in patients without the endpoint — Average temporal evolution of hs-CRP in patients without the endpoint

Figure 3 Average evolution of hs-CRP levels in patients with ACHD with and without the endpoint. All measurements taken after the endpoints were discarded. Time point zero is denoted as the time of study inclusion. The average evolution is accompanied by the $95 \%$ prediction band (thin lines). ACHD, adult congenital heart disease; hs-CRP, high-sensitivity $C$ reactive protein; MACE, major adverse cardiovascular event. 
Table 3 Association between repeated hs-CRP levels and endpoints, adjusted for clinical characteristics and (repeated) NT-proBNP and hs-TnT

\begin{tabular}{|c|c|c|c|c|}
\hline & \multicolumn{2}{|l|}{ Death or HF $(n=69)$} & \multicolumn{2}{|l|}{ Any MACE $(n=228)$} \\
\hline & $\mathrm{HR}(95 \% \mathrm{Cl})$ & $P$ value & $\mathrm{HR}(95 \% \mathrm{Cl})$ & $P$ value \\
\hline Repeated hs-CRP, mg/L (unadjusted) & 2.14 (1.73 to 2.73$)$ & $<0.001$ & 1.22 (1.10 to 1.37$)$ & $<0.001$ \\
\hline Adjusted for baseline characteristics* & 1.77 (1.38 to 2.31$)$ & $<0.001$ & $1.08(0.96$ to 1.22$)$ & 0.230 \\
\hline Adjusted for baseline NT-proBNP & $1.68(1.35$ to 2.10$)$ & $<0.001$ & 1.05 (0.94 to 1.18$)$ & 0.397 \\
\hline Adjusted for baseline characteristics and baseline NT-proBNP & 1.60 (1.26 to 2.05$)$ & $<0.001$ & $1.03(0.92$ to 1.16$)$ & 0.585 \\
\hline \multicolumn{5}{|l|}{ Repeated hs-CRP and NT-proBNP } \\
\hline Repeated hs-CRP, mg/L & $1.53(1.20$ to 2.00$)$ & 0.002 & 0.99 (0.88 to 1.12$)$ & 0.916 \\
\hline Repeated NT-proBNP, pmol/L & 2.48 (2.04 to 2.98$)$ & $<0.001$ & $1.62(1.48$ to 1.76$)$ & $<0.001$ \\
\hline \multicolumn{5}{|l|}{ Repeated hs-CRP and hs-TnT } \\
\hline Repeated hs-CRP, mg/L & $1.94(1.52$ to 2.50$)$ & $<0.001$ & $1.13(1.00$ to 1.27$)$ & 0.044 \\
\hline Repeated hs-TnT, ng/mL & 2.46 (1.93 to 3.15$)$ & $<0.001$ & 1.57 (1.38 to 1.78$)$ & $<0.001$ \\
\hline \multicolumn{5}{|l|}{ Repeated hs-CRP, hs-TnT and NT-proBNP } \\
\hline Repeated hs-CRP, mg/L & 1.54 (1.24 to 1.98$)$ & $<0.001$ & 1.00 (0.89 to 1.12$)$ & 0.984 \\
\hline Repeated hs-TnT, ng/mL & 1.19 (0.85 to 1.57$)$ & 0.248 & 1.11 (0.96 to 1.28$)$ & 0.146 \\
\hline Repeated NT-proBNP, pmol/L & 2.20 (1.74 to 2.86$)$ & $<0.001$ & $1.53(1.39$ to 1.69$)$ & $<0.001$ \\
\hline
\end{tabular}

HRs are expressed per twofold higher biomarker level.

*Age, sex, congenital diagnosis, NYHA, cardiac medication, systemic ventricular function.

HF, heart failure; hs-CRP, high-sensitivity C reactive protein; hs-TnT, high-sensitivity troponin T; MACE, major adverse cardiac event; NT-proBNP, N-terminal pro B type natriuretic peptide.

Data on whether inflammation are a bystander or a mediator in cardiovascular diseases, in general, remain controversial. ${ }^{19}{ }^{20}$ In pulmonary arterial smooth muscle cells, known for their role in $\mathrm{PAH}$, it was shown that impediment of inflammation by atorvastatin, reduces hs-CRP-induced expression of inflammatory pathways, ${ }^{21}$ suggesting causality. Unfortunately, no specific data on ACHD exist. Longitudinal hs-CRP patterns now showed that inflammation increased in patients with ACHD prior to deceasing or HF. Similar patterns have been observed in patients with chronic $\mathrm{HF}^{22}$ This at least suggests that progression of HF is associated with increasing inflammatory levels and that stable inflammatory levels are indicative of a stable cardiac status.

\section{Value of hs-CRP as prognostic biomarker in ACHD}

There may be several explanations for the independent prognostic value of hs-CRP. First, hs-CRP may reflect other processes of deterioration in ACHD that are not captured by clinical characteristics and biomarkers measured in our study, this makes causal inferences on inflammation and cardiac deterioration difficult, as this requires measurement and adjustment for all other potential confounders. Residual confounding could distort the association between hs-CRP and endpoints, resulting in false conclusions about the relation between inflammation and cardiac deterioration. Nevertheless, hs-CRP can then still be valuable for risk stratification. As hs-CRP is a non-specific inflammatory marker, hs-CRP may identify vulnerable patients in general, and therefore have prognostic value.

The biological variation of hs-CRP found in this study was considerable, which is in line with previous studies. ${ }^{23}{ }^{24}$ However, both within-subject and between-subject variation were higher and thus the corresponding RCV. This can most likely be explained by differences in study design: less frequent sampling, less hs-CRP measurements per patient and a different study population.

\section{Clinical implications}

Hs-CRP is able to provide prognostic value not captured by other prognosticators. In the absence of another likely cause of inflammation, an elevated hs-CRP ( $>3 \mathrm{mg} / \mathrm{L})$ may help to detect patients at higher risk of HF or mortality. Hs-CRP may specifically be useful to detect vulnerable patients in copresence of an elevated NT-proBNP. Measuring hs-CRP should therefore be considered in addition to the current practice of NT-proBNP in patients with ACHD. Repeated NT-proBNP can be combined with repeated hs-CRP to further enhance risk stratification. Increasing hs-CRP levels over time can identify high-risk patients who require closer monitoring, while stable levels can identify low-risk patients who can be reassured and less frequently monitored. Nonetheless, the substantial biological variation and high RCV should be kept in mind when interpreting repeated hs-CRP measurements.

If hs-CRP is induced by low-grade inflammation of an extracardiac origin, that subsequently leads to cardiac deterioration, inflammation in general may have substantial negative influences on the prognosis of patients with ACHD. For clinical practice, this would indicate that attention should be paid to inflammatory diseases in ACHD, as this might trigger HF.

Last, inflammation may be a potential therapeutic target; however, further research is needed to elucidate the inflammatory mechanism in ACHD.

\section{Limitations}

We were not able to definitely rule out concomitant inflammatory processes which may have led to increasing hs-CRP levels. The association found in our study might therefore be interfered by occurrence of acute infections in some patients. However, we included stable patients with ACHD in who hs-CRP was measured during regular study visits, making the interference of infections with their prognosis and biomarker measurement very unlikely. Also, documented baseline chronic infections and inflammatory conditions were rare; 10 patients carried a rheumatologic diagnosis, 2 patients inflammatory bowel disease, 1 latent tuberculosis and 1 cystic fibrosis. However, we had no data on depression, while depression has been associated with inflammation and poorer prognosis in ACHD. ${ }^{25}$

We could not adjust for oral contraceptive or hormonal replacement use. This may have biased the associations, as 


\section{Key messages}

\section{What is already known on this subject?}

- In clinically stable patients with adult congenital heart disease (ACHD), N-terminal pro B type natriuretic peptide (NT-proBNP) can be used to identify patients with a poor prognosis. Recently, C-reactive protein (CRP) has been associated with adverse cardiac outcomes in ACHD; however, its additive prognostic value beyond NT-proBNP remains unknown.

\section{What might this study add?}

- CRP can help to further discriminate between patients at low or high risk of death or heart failure. Higher CRP is associated with an increased risk of death or heart failure, independent of clinical characteristics, NT-proBNP and troponin T. Moreover, annual CRP measurements show that CRP levels increase prior to the occurrence of death or heart failure and repeated CRP measurements provide prognostic information beyond repeated NT-proBNP and troponin T measurements.

\section{How might this impact on clinical practice?}

- In the routine clinical assessment of patients with ACHD, an annual CRP measurement should be considered in addition to an annual NT-proBNP measurement to improve risk stratification. Further research should investigate how lowgraded inflammation is related to worsening heart failure in patients with ACHD.

hormonal replacement therapy is known to be associated with higher hs-CRP levels ${ }^{26}$ and it can be questioned whether oral contraception use could have increased the risk of certain cardiovascular events in women. Peak $\mathrm{VO}_{2}$ and peak heart rate have been associated with increased hs-CRP levels, ${ }^{7}$ as we did not have exercise data, we could not assess the prognostic value of hs-CRP beyond these variables.

\section{Conclusion}

In clinically stable patients with ACHD, an elevated hs-CRP in combination with an elevated NT-proBNP is associated with an increased risk of death or HF. Hs-CRP levels increased over time prior to the occurrence of HF or death, and serially repeated hs-CRP measurements provided additional prognostic information over repeated NT-proBNP measurements. This study shows that hs-CRP is a potential biomarker for risk stratification in patients with ACHD.

Contributors All of the authors contributed to one or more of the following: conception of design of the work (VJMB, AEvdB, JAE, JAAEC, JWR-H, EB), data collection (LWG, VJMB, AEvdB, JAE, RMK, JAAEC, JWR-H), data analysis and interpretation (LWG, JWR-H, EB), drafting the manuscript (LWG, VJMB, JWR-H, EB) and critical revision of the manuscript (all authors). All authors provided final approval of the manuscript. LWG and EB are responsible for the overall content as guarantors.

Funding Roche diagnostics provided C-reactive protein assays free of charge to support this investigator initiated study.

Competing interests None declared.

Patient consent for publication Not required.

Ethics approval The study protocol was approved by the local medical ethics committee.

Provenance and peer review Not commissioned; externally peer reviewed.

Data availability statement Data are available on reasonable request.
Supplemental material This content has been supplied by the author(s). It has not been vetted by BMJ Publishing Group Limited (BMJ) and may not have been peer-reviewed. Any opinions or recommendations discussed are solely those of the author(s) and are not endorsed by BMJ. BMJ disclaims all liability and responsibility arising from any reliance placed on the content. Where the content includes any translated material, BMJ does not warrant the accuracy and reliability of the translations (including but not limited to local regulations, clinical guidelines, terminology, drug names and drug dosages), and is not responsible for any error and/or omissions arising from translation and adaptation or otherwise.

Open access This is an open access article distributed in accordance with the Creative Commons Attribution Non Commercial (CC BY-NC 4.0) license, which permits others to distribute, remix, adapt, build upon this work non-commercially, and license their derivative works on different terms, provided the original work is properly cited, appropriate credit is given, any changes made indicated, and the use is non-commercial. See: http://creativecommons.org/licenses/by-nc/4.0/.

\section{ORCID iDs}

Annemien E van den Bosch http://orcid.org/0000-0002-0422-9860

Jolien W Roos-Hesselink http://orcid.org/0000-0002-6770-3830

Eric Boersma http://orcid.org/0000-0003-1159-7802

\section{REFERENCES}

1 van der Linde D, Konings EEM, Slager MA, et al. Birth prevalence of congenital heart disease worldwide: a systematic review and meta-analysis. J Am Coll Cardiol 2011;58:2241-7.

2 Tutarel 0, Kempny A, Alonso-Gonzalez R, et al. Congenital heart disease beyond the age of 60: emergence of a new population with high resource utilization, high morbidity, and high mortality. Eur Heart J 2014;35:725-32.

3 Khairy P, Aboulhosn J, Gurvitz MZ, et al. Arrhythmia burden in adults with surgically repaired tetralogy of Fallot: a multi-institutional study. Circulation 2010;122:868-75.

4 Cuypers JAAE, Eindhoven JA, Slager MA, et al. The natural and unnatural history of the mustard procedure: long-term outcome up to 40 years. Eur Heart $J$ 2014;35:1666-74.

5 Diller G-P, Kempny A, Alonso-Gonzalez R, et al. Survival prospects and circumstances of death in contemporary adult congenital heart disease patients under follow-up at a large tertiary centre. Circulation 2015;132:2118-25.

6 Stout KK, Broberg CS, Book WM, et al. Chronic heart failure in congenital heart disease: a scientific statement from the American heart association. Circulation 2016;133:770-801.

7 Opotowsky AR, Valente AM, Alshawabkeh L, et al. Prospective cohort study of C-reactive protein as a predictor of clinical events in adults with congenital heart disease: results of the Boston adult congenital heart disease Biobank. Eur Heart J 2018;39:3253-61.

8 Eindhoven JA, van den Bosch AE, Ruys TPE, et al. N-Terminal pro-B-type natriuretic peptide and its relationship with cardiac function in adults with congenital heart disease. J Am Coll Cardiol 2013;62:1203-12

9 Baggen VJM, van den Bosch AE, Eindhoven JA, et al. Prognostic value of $\mathrm{N}$-termina pro-B-type natriuretic peptide, troponin-T, and growth-differentiation factor 15 in adult congenital heart disease. Circulation 2017;135:264-79.

10 Baumgartner $\mathrm{H}$, Bonhoeffer P, De Groot NMS, et al. Esc guidelines for the management of grown-up congenital heart disease (new version 2010). Eur Heart $J$ 2010;31:2915-57.

11 Stout KK, Daniels CJ, Aboulhosn JA, et al. AHA/ACC guideline for the management of adults with congenital heart disease: a report of the American College of Cardiology/ American heart association Task force on clinical practice guidelines. J Am Coll Cardiol 2018;2019:e81-192.

12 Rizopoulos D. Dynamic predictions and prospective accuracy in joint models for longitudinal and time-to-event data. Biometrics 2011;67:819-29.

13 Rizopoulos D, Ghosh P. A Bayesian semiparametric multivariate joint model for multiple longitudinal outcomes and a time-to-event. Stat Med 2011;30:1366-80.

14 Scognamiglio G, Kempny A, Price LC, et al. C-Reactive protein in adults with pulmonary arterial hypertension associated with congenital heart disease and its prognostic value. Heart 2014;100:1335-41.

15 Miyamoto K, Takeuchi D, Inai K, et al. Prognostic value of multiple biomarkers for cardiovascular mortality in adult congenital heart disease: comparisons of single-/twoventricle physiology, and systemic morphologically right/left ventricles. Heart Vesse/s 2016:31:1834-47.

16 Carrick-Ranson G, Hastings JL, Bhella PS, et al. Effect of healthy aging on left ventricular relaxation and diastolic suction. Am J Physiol Heart Circ Physiol 2012;303:H315-22.

17 Eindhoven $\mathrm{JA}$, van den Bosch $\mathrm{AE}$, Jansen PR, et al. The usefulness of brain natriuretic peptide in complex congenital heart disease: a systematic review. J Am Coll Cardiol 2012;60:2140-9.

18 Rybicka J, Dobrowolski P, Lipczyńska M, et al. High sensitivity troponin T in adult congenital heart disease. Int J Cardiol 2015;195:7-14. 
19 Bisoendial RJ, Boekholdt SM, Vergeer M, et al. C-Reactive protein is a mediator of cardiovascular disease. Eur Heart J 2010;31:2087-91.

20 Anand SS, Yusuf S. C-Reactive protein is a bystander of cardiovascular disease. Eur Heart J 2010;31:2092-6.

21 Li J, Li J-J, He J-G, et al. Atorvastatin decreases C-reactive protein-induced inflammatory response in pulmonary artery smooth muscle cells by inhibiting nuclear factor-kappaB pathway. Cardiovasc Ther 2010;28:8-14.

22 van Boven N, Battes LC, Akkerhuis KM, et al. Toward personalized risk assessment in patients with chronic heart failure: detailed temporal patterns of NT-proBNP, troponin T, and CRP in the Bio-SHiFT study. Am Heart J 2018;196:36-48.
23 Macy EM, Hayes TE, Tracy RP. Variability in the measurement of C-reactive protein in healthy subjects: implications for reference intervals and epidemiological applications. Clin Chem 1997;43:52-8.

24 van den Berg VJ, Umans VAWM, Brankovic M, et al. Stabilization patterns and variability of hs-CRP, NT-proBNP and ST2 during 1 year after acute coronary syndrome admission: results of the BIOMArCS study. Clin Chem Lab Med 2020. doi:10.1515/ cclm-2019-1320. [Epub ahead of print: 08 May 2020].

25 Carazo MR, Kolodziej MS, DeWitt ES, et al. Prevalence and prognostic association of a clinical diagnosis of depression in adult congenital heart disease: results of the Boston adult congenital heart disease Biobank. J Am Heart Assoc 2020;9:e014820.

26 Ford ES, Giles WH, Mokdad AH, et al. Distribution and correlates of C-reactive protein concentrations among adult US women. Clin Chem 2004;50:574-81. 